\title{
Homotopy Perturbation Method for Boundary Value Problems with Delay Differential Equations
}

\author{
Hamood. M. Yousef* and A. I. B. MD. Ismail
}

School of Mathematical Sciences, Universiti Sains Malaysia, 11800 USM, Pulau Pinang

\begin{abstract}
This study uses the Homotopy Perturbation Method (HPM) to solve the second order of the boundary value problem (BVP) of Delay Differential Equations (DDEs). The aim of this paper to obtain the approximate solution for BVP by using the HPM with Pade approximation. Then compare the results obtained with the exact solution. A set of examples to illustrate the efficiency of the method. HPM provides an approximate analytical solution in the form of a convergent series.
\end{abstract}

Keywords: Delay Differential Equations, Boundary Value Problems, Homotopy Perturbation Method, Approximate solution, Padé approximation, Convergence of Method

\section{INTRODUCTION}

Recently, there are many problems in science, engineering, physics and biology which can be described by differential equation (DEs). Approximation analytical methods such as Variational Iteration Method (VIM), Adomian Decomposition Method (ADM), Homotopy Perturbation Method (HPM) and Homotopy Analysis Method (HAM) have been used to solve nonlinear DE involve BVP (He, 1999). In 1998 the HPM first proposed by Ji Huan He solves differential equations (He,2003; He, 2000). HPM applied for nonlinear Ordinary differential equation (He, 2000), Partial differential equation (He, 2004A), bifurcation (He, 2004A), Integration differential equations (He, 2004B), with Initial value problems (IVP) (He, 2005). DDEs with BVP has been investigated by the Laplace Adomian decomposition method (LADM) with the second order of BVP (Kanth et al.,2009). Aslamnoor and Mohyud presented DEs solution with BVP using HPM (Bellen, 2003). In this paper, some examples of DDEs have been solved and compared with the exact solution to illustrate the capability and reliability of this method. We have found that HPM is a powerful mathematical tool used for various DDEs. HPM, which provides an approximate solution to the ordinary and partial differential equations also DDEs with BVP.

\section{DELAY DIFFERENTIAL EQUATIONS}

In this study, DDEs were commonly involved in the mathematical formulation of physical and engineering phenomena. DDEs in mathematics are a type of differential equation in which the derivative of the unknown function at a particular time is given regarding the values of the equation at previous times. Also, DDEs except that they involve past values of variables and/or derivatives (Xiuying, 2008; Cveticanin,2006; Kuang, 1993; Nayfeh,1985; Mohyud-Din \& Aslam, 2008; Agarwal,1986). Here, we examine the general form of DDEs with BVP by considering as the following:

$$
\begin{aligned}
& y^{\prime \prime}(x)=g(x) y^{\prime}(x)+h(x) y(q x)+f(x), \\
& x>0, x \in R^{n}
\end{aligned}
$$

with Boundary condition's

$$
\mathrm{y}(0)=a, \mathrm{y}(1)=b \text {. }
$$


where $g(\mathrm{x}), h(\mathrm{x})$ and $f(x)$ are an analytical function $0<q<1$ and $\mathrm{a}, \mathrm{b}$ are constant.

\section{PADÉ APPROXIMATION}

In this thesis, the Padé approximation is used to provide a better estimate of the function than truncating. Padé approximation is used extensively in computer calculations. The polynomial in the denominator allows an approximation of functions that have rational singularities that can be thought of as a generalization of a Taylor polynomial (Chisholm,1973). Because these functions only use the elementary arithmetic operations, they are easy to evaluate numerically. A Padé approximate is that rational function whose power series expansion agrees with a prescribed power series to the highest possible order.

Definition.1 The Padé approximation is a rational of two polynomials $P_{L}(r)$ and $Q_{M}(r)$ in degree $N=L+M+1$. It is denoted by $[\mathrm{L} / \mathrm{M}] \mathrm{u}(\mathrm{r})$ for an analytical function with Taylor or Maclaurin's expansion, constructed from the coefficients of the series expansion of a function (George $\mathrm{Jr}$, 1975). Let assume that $u(r)$ an analytical function represents as a Taylor expansion $u(r)=\sum_{i=1}^{\infty} u_{i} r^{i}$ it has a truncated to m terms $P_{m}(r)$

$$
u(r)-b_{L}(r)=O\left(r^{L+1}\right)
$$

Then Padé approximant $R_{L / M}(r)$ can be written as follows

$$
R_{L / M}(r)=\left[\frac{L}{M}\right] u(r)=\frac{P_{L}(r)}{Q_{M}(r)}-o\left(r^{L+M+1}\right)(4)
$$

which we denote by powerful series can be transformed into Padé series is defined as we ignore $O\left(r^{L+1}\right)$

$$
\left[\frac{L}{M}\right] u(r)=\frac{P_{L}(r)}{Q_{M}(r)}=\frac{\sum_{i=0}^{L} p_{i} r^{i}}{\sum_{j=0}^{M} q_{j} r^{j}} \text { (5) }
$$

We set $q_{0}=1$ it is becoming,

$$
a_{0}+a_{1} r+a_{2} r^{2}+\ldots=\frac{p_{0}+p_{1} r \ldots+p_{M} r^{L}}{1+q_{1} r \ldots+q_{L} r^{M}}
$$

Multiply both sides of Eq.(6)by the to the denominator of the coefficients $p_{L}$ and $q_{M}$ where

$$
\begin{aligned}
& P_{0}=a_{l}, \\
& P_{l}=q_{l} a_{0}+a_{l}, \\
& \vdots \\
& P_{n}=q_{m} a_{n-m}+\cdots+q_{1} a_{n-1}+a_{m},
\end{aligned}
$$

In this paper, Padé approximation is obtained by the use of Mathematica software of various orders. Solving the linear Eq. (6), we have to calculate approximate such as [2/2], [3/3], and [4/4], ....(Baker et al., 1996).

\section{HOMOTOPY PERTURBATION METHOD}

The aim of this section is to obtain the approximate solution using the HPM for solving the second order BVP involving DDEs and compare the results obtained with the exact solution. To illustrate HPM, we consider the following general type of DE:

$$
D(y)-g(r)=0, r \in \Omega,(8)
$$

with the boundary conditions

$$
B\left(y, \frac{\partial y}{\partial n}\right)=0, \quad r \in \Gamma,(9)
$$

where $D$ is a general differential operator, it can be divided into linear parts $L$ and $N$ nonlinear term of Eq. (8) $B$ a boundary operator, $g(r)$ and a known analytical function and $\Gamma$ is the boundary of the domain $\Omega$.Can be rewritten as:

$$
L(y)+N(y)-g(r)=0,(10)
$$

By HPM, construct a Homostopy $v(r, q): \Omega \times[0,1] \rightarrow R$, which satisfies:

$H(v, q)=(1-q)\left[L(v)-L\left(v_{0}\right)\right]+q[A(v)-g(r)]=0, \quad(11)$ or

$H(v, q)=L(v)-L\left(v_{0}\right)+q L\left(v_{0}\right)+q[N(v)-g(r)]=0$

where $q \in[0,1]$ is an embedding parameter, $v_{0}$ is an initial condition, which satisfies the boundary conditions. From 
Eq. (11) or Eq. (12) we will have

$$
\begin{aligned}
& H(v, 0)=L(v)-L\left(v_{0}\right)=0, \\
& H(v, 1)=D(v)-g(r)=0 .
\end{aligned}
$$

We assume that the solution of Eq. (112) can be written as a power series in $q$

$$
v(x)=v_{0}(x)+q v_{1}(x)+q^{2} v_{2}(x)+q^{3} v_{3}(x)+\cdots,(14)
$$

Moreover, setting $q=1$ obtained the approximate solutions follows:

$$
\begin{aligned}
y & =\lim _{q \rightarrow 1} v=\lim _{q \rightarrow 1}\left(v_{0}+q v_{1}+q^{2} v_{2}+q^{3} v_{3}+\cdots\right) \\
& =\sum_{j=0}^{\infty} v_{j},
\end{aligned}
$$

The series (2) is convergent for most cases and the rate of convergence depends on $\mathrm{A}(\mathrm{v})$ (He, 2006). Using the Pade approximation for Eq. (15) by definition section (III)

$$
u_{n}(x)=\left[\frac{L}{M}\right](16)
$$

\section{NUMERICAL EXAMPLES}

In this section, we will apply the HPM on two examples involving second order DDEs with the second order BVP that have been solved by ADM and VIM. We present two examples to show the efficiency and high accuracy of the present method, camper errors the solution obtained by HPM and exact solution.

Example .1 Consider the linear DDEs with BVP as the following

$$
u "(x)=0.5 u(x)+e^{-0.5 x}\left(u_{0}(x) / 2\right)-2 e^{-x},(17)
$$

the boundary conditions

$$
u(0)=0, u(1)=e^{-1} \cdot(18)
$$

The analytical solution

$$
u(\mathrm{x})=x e^{-x},(19)
$$

According to the HPM and using Eq. (11-14), we have the solutions we construct a homotopy as follows:

$$
(1-q)\left(\frac{\partial^{2} u}{\partial x^{2}}-2 e^{-x}\right)+q\left(\frac{\partial^{2} u}{\partial x^{2}}-0.5 u+e^{-0.5 x}\left(u_{n}(.5 x)\right)\right)=0,(20)
$$

Assume the solution of Eq. (17) has the form

$$
\begin{aligned}
u(x) & =u_{0}(x)+q u_{1}(x)+q^{2} u_{2}(x)+q^{3} u_{3}(x)+\cdots \\
& =\sum_{j=0}^{\infty} q^{j} u_{j}(x)
\end{aligned}
$$

where $u_{j}(x)$ are functions can be determined.

$$
\begin{aligned}
& q^{0}: \frac{\partial^{2} u_{0}}{\partial x^{2}}=\frac{\partial u_{0}}{\partial x}, \quad u_{0}(0)=0,(22) \\
& q^{1}: \frac{\partial^{2} u_{1}}{\partial x^{2}}=0.5 u-e^{-0.5 x} u_{n}(.5 x) \\
& u_{1}(0)=0 \\
& q^{4}: \frac{\partial^{2} u_{4}}{\partial x^{2}}=0.5 u-e^{-0.5 x} u_{n}(.5 x) \\
& u_{4}(0)=0
\end{aligned}
$$

where

$$
u_{k+1}(0)=0, k \geq 1 \text { considering }
$$$$
u_{0}(x)=u(0)=f(x) \text {, we have }
$$

$$
u(0)=u_{0}(x)=0,(25)
$$

Using Pade approximation for Eq. (22-24) by definition

$$
u_{n}(x)=\left[\frac{L}{M}\right]=x-x^{2}+\frac{x^{3}}{2}+\frac{x^{4}}{6}(26)
$$

Table 1. Comparisons of HPM with an exact solution by 4-termsfor Example. (1)

\begin{tabular}{cccc}
\hline$x$ & Exact solution & Approximate [ HPM] & Absolute Error \\
\hline 0 & 0 & $-3.410605131648481 \times 10^{-13}$ & $3.410605131648481 \times 10^{-13}$ \\
0.2 & 0.26812801841425576 & 0.2681280184139023 & $3.534394998894186 \times 10^{-13}$ \\
0.4 & 0.35946317129377725 & 0.3594631711375307 & $1.562465712368066 \times 10^{-10}$ \\
0.6 & 0.3614330542946425 & 0.36143304174098034 & $1.255366216534881 \times 10^{-8}$ \\
0.8 & 0.3230344287914486 & 0.32303415205801045 & $2.767334381648112 \times 10^{-7}$ \\
1 & 0.2706705664732254 & 0.27066756119533864 & $3.0052778867695196 \times 10^{-6}$ \\
\hline
\end{tabular}




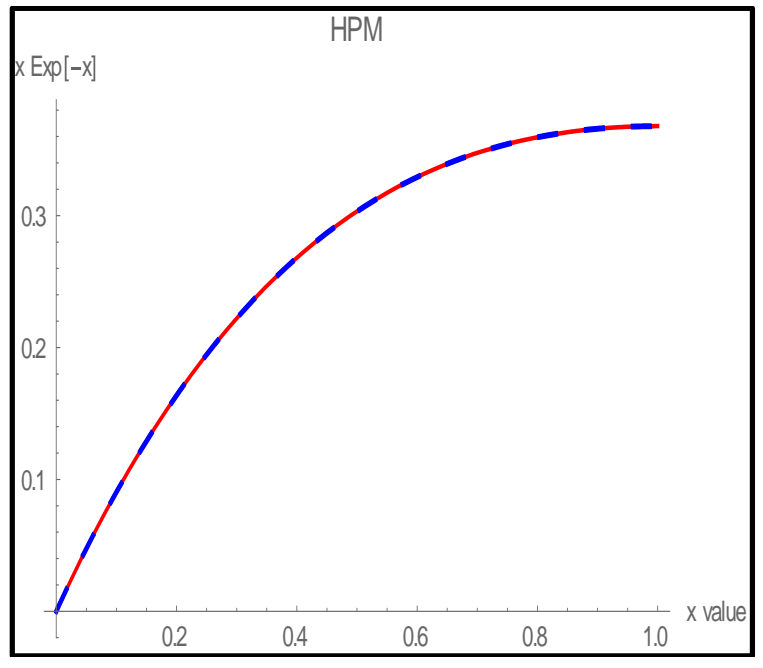

Figure 1. Absolute errors for HPMs with Exact solution four terms of example (1)

The comparison between the approximate solutions obtained by HPM and the exact solution was given in table (1) and figure (1) it shows the graphical representation the approximate solutions is very close to the exact solution.

Example.2 Consider the linear DDEs with following

$\frac{d^{2} u}{d x^{2}}=1+2\left(1+\frac{1}{8} x^{2}\right) \cos \left(\frac{1}{2} x\right)-2 \cos \left(\frac{1}{2} x\right) u\left(\frac{1}{2} x\right)$ $\mathrm{x} \in\left[0, \frac{\pi}{4}\right]$

subject to the boundary conditions

$$
u(0)=1, u\left(\frac{\pi}{4}\right)=1+\frac{\sqrt{2}}{2}+\frac{\pi^{2}}{32} .(28)
$$

The analytical solution

$$
u(x)=1+\frac{1}{2} x^{2}+\sin (x)(29)
$$

According to the HPM, the construction of homotopy is as follows

$$
\begin{aligned}
& (1-q)\left(\frac{\partial^{2} u}{\partial x^{2}}-1\right)+ \\
& q\left(\frac{\partial^{2} u}{\partial x^{2}}-1-2\left(1+\frac{x^{2}}{8}\right) \cos \left(\frac{x}{2}\right)+2 \cos \left(\frac{x}{2}\right) u\left(\frac{x}{2}\right)\right)=0
\end{aligned}
$$

Assume the solution of Eq. (19) has the form

$$
\begin{aligned}
u(x) & =u_{0}(x)+q u_{1}(x)+q^{2} u_{2}(x)+q^{3} u_{3}(x)+\cdots \\
& =\sum_{j=0}^{\infty} q^{j} u_{j}(x)
\end{aligned}
$$

where $u_{j}(x)$ are functions yet to be determined., we have

$$
u_{n}(x)=\left[\frac{L}{M}\right]=1+x+\frac{x^{2}}{2}-\frac{x^{3}}{6}+\frac{x^{5}}{120}(32)
$$

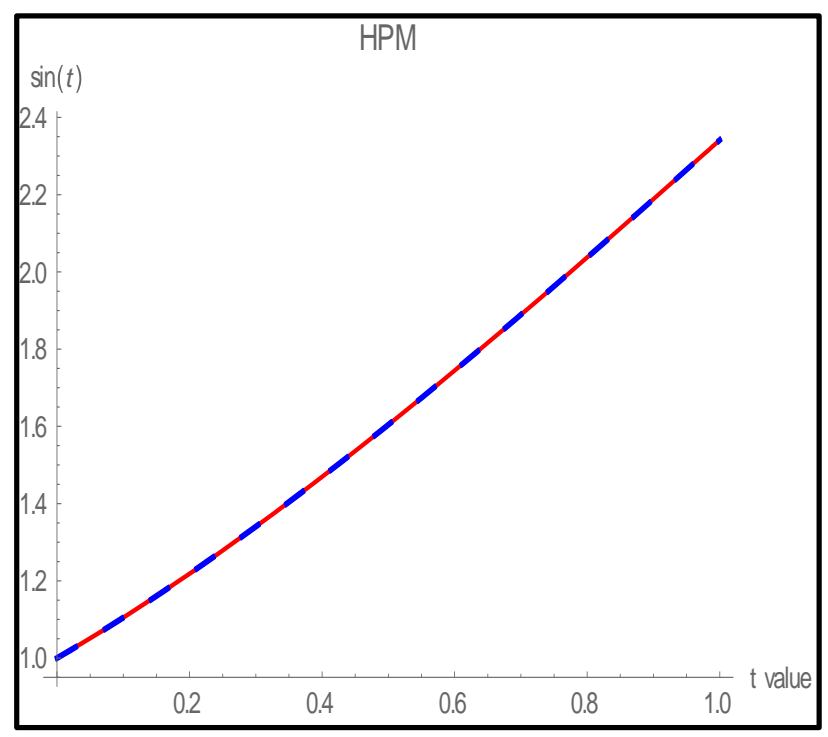

Figure 2. The errors between results obtained using HPM and the exact solution with four terms.

Table 2. Comparisons of HPM with an exact solution by 4-terms for Example. (2)

\begin{tabular}{cccc}
\hline$x$ & Analytical solution & Approximate [HPM] & Absolute Error \\
\hline 0 & 0 & $-3.410605131648481 \times 10^{-13}$ & $3.410605131648481 \times 10^{-13}$ \\
0.2 & 0.26812801841425576 & 0.2681280184139023 & $3.534394998894186 \times 10^{-13}$ \\
0.4 & 0.35946317129377725 & 0.3594631711375307 & $1.562465712368066 \times 10^{-10}$ \\
0.6 & 0.3614330542946425 & 0.36143304174098034 & $1.255366216534881 \times 10^{-8}$ \\
0.8 & 0.3230344287914486 & 0.32303415205801045 & $2.767334381648112 \times 10^{-7}$ \\
1 & 0.2706705664732254 & 0.27066756119533864 & $3.0052778867695196 \times 10^{-6}$ \\
\hline
\end{tabular}


The previous results show that the HPM is efficient and simple through a few terms of the series that used to get the approximate solutions.

\section{CONVERGENCE OF HPM}

We introduce the study of the problem of convergence of the HPM.

Theorem.1 Let B a Banach space.

a- $\quad \sum_{i=0}^{\infty} v_{i}$ obtained by (18), convergence to B, if $\sum_{i=0}^{\infty} v_{i}$,
satisfies in
b- $\quad S=L^{-1} N\left(s+v_{0}\right)+u_{0}+L^{-1}(f(t))$

Proof. (a) we have

$$
\begin{gathered}
\forall v, \bar{v} \in X ;\left\|S_{n+1}(t)-S_{n}(t)\right\| \leq r\|v-\bar{v}\|, 0<r<1 . \\
\begin{aligned}
\left\|S_{n+1}-S_{n}\right\|= & \left\|u_{n+1}(t)\right\| \leq r\left\|u_{n}(t)\right\| \leq r^{2}\left\|u_{n-1}(t)\right\| \leq \cdots \\
& \leq r^{n+1}\left\|u_{0}(t)\right\|
\end{aligned}
\end{gathered}
$$

For any, $n . m \in N, n \geq m$ we drive

$$
\begin{aligned}
\left\|\varphi_{\mathrm{n}+1}-\varphi_{n}\right\|=\| & \left(\varphi_{\mathrm{n}+1}-\varphi_{n}\right)+\left(\varphi_{\mathrm{n}+1}-\varphi_{n}\right)+\cdots \\
& +\left(\varphi_{\mathrm{n}+1}-\varphi_{n}\right) \| \\
& \leq\left\|\varphi_{\mathrm{n}+1}-\varphi_{n}\right\|+\left\|\varphi_{\mathrm{n}+1}-\varphi_{n}\right\|
\end{aligned}
$$

and

$$
\begin{aligned}
\left\|\varphi_{\mathrm{n}+1}-\varphi_{n}\right\| \leq & \left\|\left(\varphi_{n}-\varphi_{n-1}\right)\right\|+\left\|\left(\varphi_{n-1}-\varphi_{n-2}\right)\right\|+\cdots \\
& +\left\|\left(\varphi_{m+1}-\varphi_{m}\right)\right\| \\
\leq & r\left\|\varphi_{n}\right\|+r^{2}\left\|\varphi_{n-1}\right\|+\cdots+r^{n+1}\left\|\varphi_{0}\right\| \quad(34) \\
\leq & \left(r^{m+1}+r^{m+2}+\cdots\right)\left\|\varphi_{0}\right\| \\
& =\frac{1-r^{n-m}}{1-r}\left\|\varphi_{0}\right\|
\end{aligned}
$$

So

$$
\lim _{n, m \rightarrow \infty}\left\|\left(V_{n}-u\right)\right\|=0 \text {, }
$$

Therefore, $\left\{S_{n}\right\}_{n=0}^{\infty}$ a Cauchy sequence in Banash space and it is convergent.

\section{CONVERGENCE OF HPM}

The HPM convergence to the exact result is studied in this section. The series solution $u(x)=\lim _{p \rightarrow 1} v(x)=v+v_{0}+v_{1}+v_{2}+\cdots$ obtained by HPM converge to the exact solution. The homotopy standard for Eq. (2) has a scheme

$$
\begin{aligned}
& u_{0}=u_{0} \\
& u_{1}=L^{-1}\left(f(x)-u_{0}\right) \\
& u_{2}=-L^{-1}\left(N_{1}(x)\right) \\
& \vdots \\
& u_{n+1}=-L^{-1}\left(N_{n}(x)\right)
\end{aligned}
$$

Theorem.1 (Ayati \& Biazar,, 2015)Let $A$ be a Banach space. The series solution $\sum_{m=1}^{+\infty} u_{m}(t)$ converges to the exact solution $s \in A$ if $\exists(0 \leq \gamma<1)$ such that $\left\|u_{n+1}\right\| \leq \gamma\left\|u_{n}\right\|, \forall n \in \square \bigcup\{0\}$.

Theorem.2(Ayati and Biazar, 2015) for every $n \in \square \bigcup\{0\}$, the parameters define as

$$
\gamma_{n}=\left\{\begin{array}{cc}
\frac{\left\|u_{n+1}\right\|}{\left\|u_{n}\right\|}, & \left\|u_{n}\right\| \neq 0 \\
0, & \left\|u_{n}\right\|=0
\end{array}\right.
$$

In Theorem .2 the DDE series solution $\sum_{m=0}^{\infty} u_{m}(t)$ converges to the exact solution, when $u_{n}(t)$. For the $0 \leq \gamma_{n} \leq 1, \forall n \in \square \bigcup\{0\}$.

\section{RATIO TEST CONVERGENCE}

The ratio test can be used to study the convergence of the approximate LTHPM. Let the sequence be as according to the above Theorem 6.2. We will study the convergence of 
HPM and HPM by using the ratio test for examining the conditions $0 \leq \gamma_{k} \leq 1, k=0,1,2,3, \cdots \quad$ for some examples of DDEs.

\section{APPLICATION OF RATIO TEST OF CONVERGENCE}

Example 1 We check the convergence of the HPM for example 1

$$
\begin{aligned}
& \gamma_{0}=\left\|\frac{u_{2}}{u_{1}}\right\|=0.3982570635154341<1 \\
& \gamma_{1}=\left\|\frac{u_{3}}{u_{2}}\right\|=0.21192630386316658<1 \\
& \gamma_{2}=\left\|\frac{u_{4}}{u_{3}}\right\|=0.13996588393175585<1
\end{aligned}
$$

Example.2 We check the convergence of the HPM for example 2

$$
\begin{aligned}
& \gamma_{0}=\left\|\frac{u_{1}}{u_{0}}\right\|=0.03622530276953514<1 \\
& \gamma_{1}=\left\|\frac{u_{2}}{u_{1}}\right\|=0.013694821336785339<1 \\
& \gamma_{2}=\left\|\frac{u_{3}}{u_{2}}\right\|=0.0074362023444989765<1
\end{aligned}
$$

where $\gamma_{1}, \gamma_{2}$ and $\gamma_{3}$ are less than one. So, the approximate HPM of DDEs are convergent.

\section{SUMMARY}

In this paper, HPM is a powerful approximate method was introduced to obtain an analytical approach for DDEs with BVPs. The scheme based HPM has been formulated to get the solution of DDEs directly without reducing into first order systems. The HPM has been successfully used to study some examples of DDEs. The HPM avoids Adomian polynomials which can be difficult in some cases. The results show that the proposed method is a more workable mathematical tool for finding the exact and approximate solutions of nonlinear equations. The study of the convergence to the exact solution for DDEs with BVP briefly addressed. The HPM is very useful and convenient method. 


\section{REFERENCES}

Agarwal, R.P., (1986). Boundary value problems for higher-order differential equations. World Scientific, Singapore.

Ayati, Z. \& Biazar, J. (2015). On the convergence of homotopy perturbation method. Journal of the Egyptian Mathematical Society, 23, 424-428.

Baker, G. A., Baker Jr, G. A., GRAVES-MORRIS, P. \& BAKER, S. S. (1996). Padé approximants, Cambridge University Press.

Bellen A, Zennaro M. (2003). Numerical methods for delay differential equations, in: Numerical Mathematics and Scientific Computation, The Clarendon Press Oxford University Press, New York, 2003.

Chisholm, J. (1973). Rational approximants defined from double power series. Mathematics of Computation, 27, 841-848.

Cveticanin, L. (2006). Homotopy Perturbation Methodfor pure nonlinear differential equation, Chaos, Solitons and Fractals 30 1221-1230.

George Jr, A. (1975). Essentials of Padé approximants, Elsevier.

He, J.H. (1999). Homotopy perturbation technique. Comput. Methods Appl. Mech. Engng., 178 (3-4): 257-262.

He, J.H. (2003). Homotopy perturbation method: a new nonlinear analytical technique. Appl. Math. Comput., 135: 73-79.

He, J.H. (2003). Homotopy perturbation method: A new nonlinear analytical technique, Applied Mathematics and Computation $135-73-79$.

He, J.H. (2004A). The Homotopy Perturbation Method for nonlinear oscillators with discontinuities. Appl. Math. Comput., 151: 287-292.

He, J.H. (2004B). Comparison of Homotopy Perturbation Method and homotopy analysis method. Appl. Math. Comput., 156: 527-539.
He, J.H. (2005) Application of Homotopy Perturbation Method to nonlinear wave equations, Chaos Solitons and Fractals, 26695-700.

He, J.H., (2000). A coupling method of a homotopy technique and a perturbation technique for nonlinear problems, International Journal of NonLinear Mechanics 35 37-43.

He, J.H., (2006). Homotopy Perturbation Method for solving boundary value problems, Physics Letters A, $350-87-88$.

He, J.H., (2000). A review of some new recently developed nonlinear analytical techniques. Int. J. Nonlinear Sci. Numer. Simul., 1 (1): 51-70.

Kanth, Ravi, A.S.V. and Aruna, K., (2009). He's homotopy-perturbation method for solving higherorder boundary value problems. Chaos \&Solitons and Fractals, 41: 1905-1909.

Kuang Y., (1993). Delay Differential Equations with Applications in Population Dynamics, Academic press, Inc., New York.

Liao, S.J. (2003). Beyond Perturbation: Introduction to the Homotopy Analysis Method. Boca Raton: CRC Press.

Liu, G.R. and Wu, T.Y. (1973). Differential quadrature solutions of eighth-order boundary-value differential problems. J. Comput. Appl. Math., 145: 223-235.

Mohyud-Din, S.T., and M. Aslam Noor, (2008). Homotopy perturbation method for solving sixthorder boundary problems. Comput. Math. Appl., 55: 2953-2972.

Nayfeh, A.H. (1985). Problems in Perturbation. John Wiley, New York.

Usman M., U. Ali, T. Zubair, I. Rashid and S. T. MohyudDin, (2012). Modified Homotopy Analysis Method for Heat and Wave-like Equations, I. J Mod. Math. Sci. 2(2), 61-69. 
Xiuying, Li. (2008). Numerical Solution of an Initial Bounday Value Problem with Non-Local Condition for the Wave Equation, Mathematical Sciences, Vol. 2. No. 3 281-292.

Yousef, H. M., \& Ismail, A. I. (2017, November). Appling Laplace Adomian decomposition method for delay differential equations with boundary value problems. In AIP Conference Proceedings (Vol. 1905, No. 1, p. 030041). AIP Publishing. 